\title{
Credibility Analysis on Evaluation of Combat Effectiveness
}

\author{
Dong Li ${ }^{a}{ }^{*}$, Guo Lei Sun ${ }^{b}$ and Xin Qi Guo ${ }^{c}$ \\ Naval Aeronautical and Astronautical University, Yantai, China \\ aLidong501@sohu.com, bzishisun@163.com, chyguoxq@163.com
}

Keywords: Operational effectiveness evaluation; Credibility analysis; Meta-appraisement; Risk control.

\begin{abstract}
There are many uncertain factors in the evaluation process of operational effectiveness evaluation. This paper mainly studies the credibility problem of operational effectiveness evaluation; analyzes the emergence of operational effectiveness evaluation of weapon equipment in the uncertain factors, the basic principles of inductive Meta-appraisement method, constructs the operational effectiveness evaluation credibility based on Meta-appraisement framework of analysis; On the basis of the above, some key problems of Meta-appraisement method were researched.
\end{abstract}

\section{Introduction}

Operational effectiveness evaluation is an important work of the weapon and equipment demonstration. The risk of operational effectiveness evaluation is from the complexity of effectiveness evaluation activities [1]. Usually the decision-making departments to accept the combat effectiveness of argument nodes default premise is: the evaluation conclusion is trustworthy and reliable. In evaluating the operational effectiveness of reality, this assumption can meet the conditions, is often difficult to determine. This implies a problem: how to end combat effectiveness evaluation of the credibility of the conclusion [2] degree? Effectiveness evaluation process is affected by many uncertain factors in the process, usually is not easy to get the assessment of reliable, stable conclusion. Credibility evaluation of operational effectiveness analysis needs to assess the higher level structural. This paper analyzes the evaluation of operational effectiveness of weapon equipment in the uncertain factors, and based on Meta-appraisement of the credibility evaluation analysis model, analysis of credibility and uses this model to evaluate the results.

\section{Summary of Meta-appraisement}

Introduce of meta-appraisement. Meta-appraisement [2,3] means to evaluate the evaluation. It is to evaluate the primary-evaluation activity and results as the object, from the overall perspective of multi reflection awareness, and then make the objective, scientific, comprehensive evaluation conclusion on its reliability and validity. Meta-appraisement has to the original evaluation deviation control function. Specific include:

The detection evaluation deviation. Through long-term development, Meta-appraisement has developed many Evaluate deviation identification methods, such as the variance method, the Kendall coefficient of concordance method [4,5], etc. Through the analysis of some of the original evaluation data and process, these methods can identify the original deviation link evaluation.

Correct appraisal deviation. By using the statistical method and other methods based on deviation identification, Meta-appraisement analysis the deviation reasons of the original assessment, calculates the deviation value, thus corrects the bias evaluation.

Feedback evaluation deviation. Meta-appraisement is by seeking and different views on an assessment or a set of evaluation to obtain a wide range of views, and then points out the existing problems in their evaluation to the main body of the original assessment, and provide constructive comments and valuable information to them, thus improving the overall quality evaluation. 
Using meta-appraisement method to analyze credibility evaluation of operational effectiveness of weapon system, the basic principles needs assessment:

The principle of objectivity. Meta-appraisement is a comprehensive method simultaneously has the theoretical property and technology property.

The principle of integrity. Meta-appraisement method to be evaluated overall object as the basic starting point.

The principle of globally. The overall principle requirements of the various factors affecting the Meta-appraisement activities can be careful inspection and weighing.

The principle of comprehensiveness. Research on comprehensive analysis is the key link of the whole process of complete element evaluation.

The credibility evaluation analysis framework based on the Meta-appraisement. Combining the meta-appraisement idea, constructs the risk assessment framework of operation, as shown in Fig. 1.

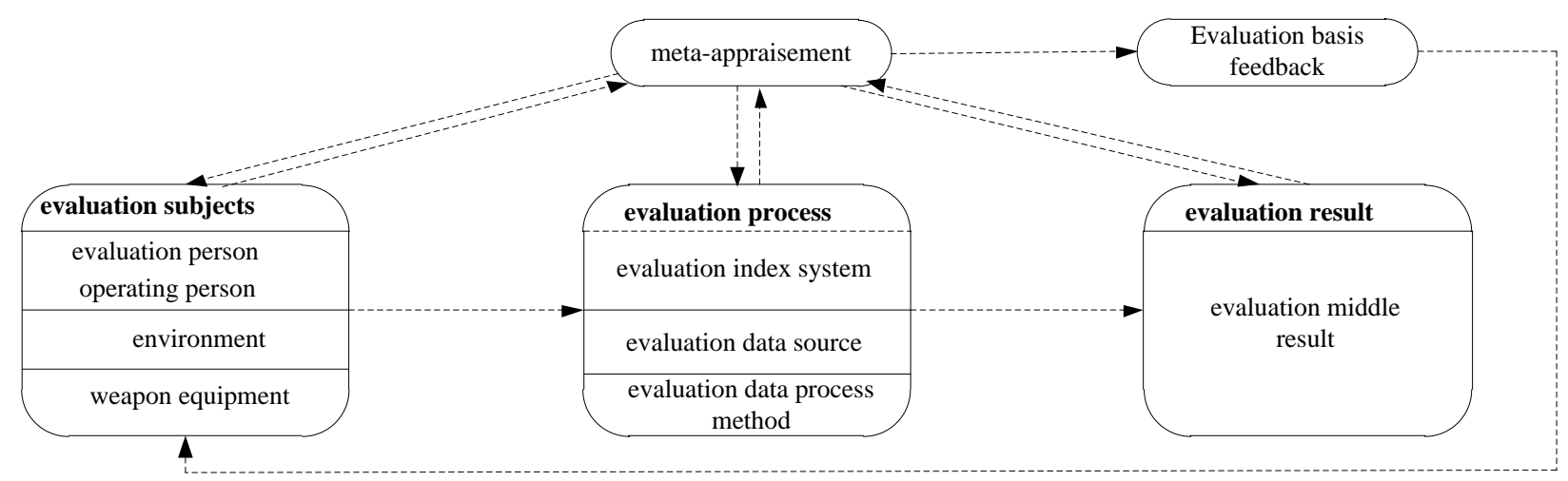

Fig. 1 Analysis framework to the credibility evaluation based on meta-appraisement

This framework gives an assessment of the credibility analysis of factors that need to be examined, and also presented the direction and main operation to reduce the evaluation risk.

\section{Analysis of evaluation results credibility based on meta-appraisement framework}

In the above credibility evaluation framework based on the meta-appraisement, evaluation deviation can be approximated as the reliability of evaluation. It is a comprehensive indicator, usually describe with uncertain factors with the evaluation process of each evaluation step.

In the evaluation process, to describe the reliability of evaluation, the main indicators of the need to consider are the evaluators credibility, evaluation index system credibility and the evaluation results credibility. Firstly the index were analyzed, and then integrated to obtain the total assessment of the reliability $\operatorname{Re} l$, such as

$\operatorname{Re} l=\operatorname{Cre}($ agent,$i d x$, res $)$

The ${ }^{C r}{ }^{(*)}$ representative evaluation factors *'s reliability metric composite function. Agent, idx, res are evaluation factors, index system and evaluation conclusion elements.

Evaluators' reliability measure. In the evaluation of operational effectiveness of weapon equipment, Evaluators mostly exist in the form of groups. To evaluate the consistency between the credibility evaluations, the main method is M.Kendall harmonious coefficient method.

The Kendall coefficient of concordance [6,7] is in accordance with the differences in evaluation index elements of the level and between the sizes, degree of consistency between views to measure multiple evaluations. The Kendall coefficient of concordance can be defined, namely

$$
w=12 S / m^{2}\left(n^{3}-n\right)
$$


In this Eq.2, $\mathrm{m}$ is assessment of the number of evaluators, and $\mathrm{n}$ for the assessment of a number of indicators, $\mathrm{S}$ is obtained from the quadratic sum by $R_{j}$ and $\bar{R}_{j}(\mathrm{j})$.

$$
S=\sum_{j=1}^{n}\left(R_{j}-\bar{R}_{j}\right)^{2}=\sum_{j=1}^{n} R_{j}^{2}-\frac{1}{n}\left(\sum_{j=1}^{n} R_{j}\right)^{2}
$$

In Eq. $3,{ }^{R}$ is the evaluation grade of the first $\mathrm{j}$ to the evaluation indicators for $\mathrm{m}$ members of the evaluation, the method of calculation $R_{j}$ is the first $\mathrm{i}$ bit rater assignment of evaluation indexes $r_{i j}$ according to the large order, if strictly monotone, might as well set ${ }^{r_{i j 1}}<r_{i j 2}<\ldots<r_{i j n} . \mathrm{j} 1, \mathrm{j} 2, \ldots$, jn is a ranking sort $(1,2, \ldots \mathrm{n})$.

Structural mapping:

$r_{i j k} \rightarrow k$

It is noted that the evaluation index grade by $\mathrm{i}$ and $\mathrm{j}$ is $k(i, j)$,

$$
R_{j}=\sum_{i=1}^{m} k(i, j) \quad(\mathrm{j}=1,2, \cdots, \mathrm{n})
$$

If not strictly monotone, the evaluator about certain evaluation index assignment has the same situation, which needs to revise this formula as following one:

$$
w=S /\left[m^{2}\left(n^{3}-n\right) / 12-m \sum_{i=1}^{m} T_{i}\right]
$$

Calculation for $T_{i}$, which is the first an evaluator for assignment of the equal condition in the measurement can formulated as:

$$
T_{i}=\frac{1}{12} \sum\left(l^{3}-l\right)
$$

Among them, the number $l$ is the occurrences of the equal value and sum is different (indicating the presence of equal value) value.

The Kendall coefficient of concordance is in general $(0,1)$ between the $(0<w<1)$. Much can be thought of as the ideal value consensus Kendall concordance coefficient, and the value of many small can be seen as a lack of consistency of opinion.

Effectiveness of index systems of operational evaluation and reliability and validity of element. (a) Meta-appraisement of index system reliability [8,9,10]. The evaluation index of reliability refers to the evaluation index values in the multiple observation results in a degree of consistency. In the meta-appraisement process, through the Cronbach internal consistency coefficient observe the evaluation index of reliability. A Cronbach coefficient calculation formula is:

$$
r_{a}=\frac{k}{k-1}\left|1-\frac{\sum S_{i}^{2}}{S^{2}}\right|
$$

In Eq.8, that $\mathrm{k}$ as index system contains index number, when the index system contains several sub index layer, $\mathrm{k}$ as sub index layer contains index number; $S_{i}$ is the standard deviation of each index, $S_{i}^{2}$ is the index variance, $\mathrm{S} 2$ is total variance.

(b) Meta-appraisement of validity of the evaluation of the index system. Validity of the Evaluation index refers to the evaluation index in the extent to which describes the assessment and reflect the characteristics of the object category assessment, the assessment indicators reflect the extent to which the accuracy of the evaluation object and objective elements. The formula of evaluation index validity or "content validity ratio"(CVR) is given by:

$$
C V R=\frac{n e-N / 2}{N / 2}
$$

In the above equation, ne is the number of evaluation which reflects the Appraisal object category, while $\mathrm{N}$ stands for the total sum. CVR turn out to be negative when $n e$ is less than $50 \%$,while CVR show result as 1 when $n e$ is $100 \%$. 
The credibility of evaluation results. The analysis of evaluation credibility requires multiple evaluation results. In RMB based on assessment of assessment framework, the required assessment is obtained through designed assessment scheme. In assessment of design scheme, focuses on the changes of input and the output, especially the changes of the evaluation results, which can reflect the changes of the trends of reliability assessment.

Based on the evaluation results of credibility evaluation model steps are build as follows:

First, select alternatives, and determine the evaluation index system.

Second, several rounds of evaluation of each alternative design experiments are designed in turn to get the result of the assessment, and the mean by each evaluation results can be obtained according to the following Eq.10.

$$
E\left(A_{i}\right)=\frac{1}{m} \sum_{k=1}^{m} R\left(A_{i k}\right)
$$

Then, deviation of the result of the experimental scheme evaluation can be obtained according to the Eq.11 to calculate.

$$
\operatorname{Var}\left(A_{i k}\right)=\xi *\left(R\left(A_{i k}\right)-E\left(A_{i}\right)\right)^{2}
$$

$\xi$ are the Risk punishment coefficients, which are related to the mean deviation of $h$, when $h$ is above zero, the deviation is diminishing, and vice versa.

$$
\xi=\left\{\begin{array}{l}
1, h: R\left(A_{i k}\right)-E\left(A_{i}\right)<0 \\
1 /(h+1), h: R\left(A_{i k}\right)-E\left(A_{i}\right)>0
\end{array}\right.
$$

Hence, the total average deviation for the alternatives $A_{i}$ :

$$
\operatorname{Var}\left(A_{i}\right)=\frac{1}{m} \sum_{k=1}^{m} \xi *\left(R\left(A_{i k}\right)-E\left(A_{i}\right)\right)^{2}=\frac{1}{m} \sum_{m}^{1} \operatorname{Var}\left(A_{i k}\right)
$$

In the same way, the final evaluation results of sample table are obtained by completion of all alternative assessment program evaluation. Finally, stability analysis of various evaluation schemes is given according to the mean value and variance of test results evaluation (bias).

In summary, stability of the evaluation results can be described by mean and deviation .According to the assessment of mean and volatility of these evaluations. Scheme can make comparison between the results of equipment scheme evaluation. Mean of the assessment reflects the efficiency evaluation, and deviation indicates possible risk assessment. In the evaluation of operational effectiveness of weapon equipment, it is necessary to best operational effectiveness value and low risk evaluation results to make reliable scheme.

\section{Summary}

It is liable to have great risks in future decision as many uncertainties lies in the effectiveness evaluation . it is necessary to have an analysis to test the risk assessment before the evaluation of weapon equipment operational effectiveness evaluation results is provided to decision makers The analysis presented in this paper is based on the meta-appraisement of operational effectiveness evaluation for the construction of credibility, measure and control risk assessment framework based on meta-appraisement, analysis of credibility in this framework is mainly discussed the evaluation result, with clear thinking, simple and practical, easy to implement on computer, equipment demonstration and other fields have a wide range of uses.

\section{References}

[1] T. Chen: Decision Analysis. (Beijing: Science Press, 1988). (In Chinese).

[2] T.F Tian. Studies in Foreign Education. Vol.41(2014) No.6, p.111-119. (In Chinese). 
[3] Sawaragi T, Suzui H Y Y: Meta-appraisement of Human-agent Interactions Using Lens Model A analysis//In SICE 2002.Proceedings of the 41st SICE Annual Conference, 2002.

[4] Stufflebeam D L. American Journal of Evaluation. Vol.(2001) No.2, p.28-31.

[5] G.S. Sun. Journal of Northwest Normal University, Natural Science Edition. Vol.30 (1994) No.1, p.110-112.

[6] Z.G Li and Q.Chen. Shandong Higher Education. Vol.(2014) No.10, p.14-19. (In Chinese).

[7] Stufflebeam and D. L. Journal of MultiDisciplinary Evaluation. Vol.15 (2011) No.7, p.99-158.

[8] Scriven and Michael. Journal of MultiDisciplinary Evaluation. Vol.11 (2009) No.6, p.3-8.

[9] Antonella Nuzzaci. Journal of US-China Public Administration. Vol.10 (2013) No.1, p.95-110.

[10]Helga C. Hedler and Namara Gibram. Journal of MultiDisciplinary Evaluation, Vol.12 (2009) No.6, p.210-223. 